\title{
Transformationspfade hin zu einer Green Economy
}

Um aktuellen Umweltproblemen effektiv zu begegnen, bedarf es einer transformativen Umweltpolitik. Das Vorhaben evolution2green zielt darauf ab, das Potenzial der deutschen Gesellschaft und Wirtschaft sowie mögliche Strategien für eine transformative Umweltpolitik und damit einhergehende politische Handlungsoptionen von allen Seiten zu beleuchten. Von Walter Kahlenborn, Linda Hölscher und Daniel Weiß

D iverse Schlagzeilen aus den letzten Monaten unterstreichen die Bedeutsamkeit des Themas Klimawandel. Immer neue Erkenntnisse zur Entwicklung des Weltklimas wechseln sich ab mit Berichten über extreme Wetterereignisse weltweit. Der im Herbst 2019 erscheinende Bericht des Weltklimarats (IPCC) zu Ozeanen und der Kryosphäre wird die neueren Erkenntnisse zum Meeresspiegelanstieg in die breite Öffentlichkeit tragen. Dabei wird deutlich werden, dass der Meeresspiegelanstieg potenziell wesentlich höher ausfällt als bisher gedacht. Gleichzeitig weisen erste Ergebnisse aus einer neuen Reihe von globalen Klimamodellen (CMIP-6) darauf hin, dass der Temperaturanstieg in diesem Jahrhundert noch stärker ausfallen könnte, als in den bisherigen Publikationen des IPCC angenommen. So könnte der sechste Sachstandsbericht die schon beunruhigenden Aussagen des letzten Berichts noch übertreffen. Parallel dazu häufen sich Berichte über extreme Wetterereignisse und deren Folgen wie etwa durch lange Dürreperioden begünstigte Waldbrände in Sibirien, Hitzewellen mit zahlreichen Todesopfern in Indien, katastrophale Zyklone und mit ihnen einhergehende Überschwemmungen in Mosambik oder eine extreme Eisschmelze in Grönland. Auch in Deutschland lenken verschiedene Wetterextreme und klimabedingte Ereignisse zunehmend den Blick auf den Klimawandel. Neue Rekorde bei Waldbränden, neue Spitzenwerte bei den Temperaturen und problematische Niedrigwasserstände zeigen uns, dass der Klimawandel auch in Deutschland bereits angekommen ist.

\section{Zeitalter der Transformationen}

Als Reaktion auf diese Entwicklungen haben sich in den letzten Jahren diverse zivilgesellschaftliche Initiativen formuliert, aktuell besonders prominent Fridays for Future. Das Ziel dieser Bewegungen ist eine kohlenstoffneutrale Wirtschaft und Gesellschaft. Wie schwierig es ist, dieses Ziel zu erreichen, zeigen derzeit wieder die intensiven Diskussionen im Zusammenhang mit einer $\mathrm{CO}_{2}$-Steuer sowie die erheblichen Defizite der bundesdeutschen Klimapolitik, wenn es um die Zielerreichung der nun bevorstehenden Ziele für 2020 geht.

Die Schwierigkeiten, dem Klimawandel in ausreichender Geschwindigkeit und mit dem entsprechenden Nachdruck zu begegnen, überdecken gelegentlich, dass die Problemlage insgesamt noch wesentlich größer ist. Nicht nur mit Blick auf das Klima testet die Menschheit die Belastbarkeit des Erdsystems derzeit aus. In zahlreichen weiteren Bereichen finden aktuell massive anthropogene Eingriffe statt. Verschiedene Konzepte, wie etwa die planetaren Leitplanken des Wissenschaftlichen Beirats der Bundesregierung Globale Umweltveränderungen (WGBU) beziehungsweise die ähnlich gelagerten "planetaren Grenzen“ (Rockström et al. 2009), versuchen dies nachzuzeichnen und wissenschaftlich zu belegen. Faktisch ist der Kenntnisstand in vielen Fällen noch deutlich geringer als dies im Bereich der Klimaerwärmung der Fall ist, gleichwohl ist es recht offenkundig, dass die Menschheit im 21. Jahrhundert zahlreiche gravierende Umweltprobleme wird meistern müssen. Dazu gehören massive Biodiversitätsverluste und ein Artensterben, wie es dies in Jahrmillionen nicht gegeben hat. Dazu gehören aber auch viel zu hohe Stickstoffeinträge, wie auch eine Verschmutzung der Landschaft und der Meere durch Plastik sowie ein ständig anwachsender Ausstoß chemischer Substanzen (UNEP 2019).

Diese Probleme müssen vor dem Hintergrund diverser Faktoren, die einer Lösung hinderlich im Wege stehen, bewältigt werden. So wird die Weltbevölkerung in den nächsten 80 Jahren voraussichtlich noch deutlich zunehmen und damit ein erheblicher weiterer Druck auf die Umwelt ausgelöst. In einer international verflochtenen und zunehmend unübersichtlichen Welt bieten gleichzeitig die Nationalstaaten immer weniger Halt und Orientierung bei der notwendigen Koordination und Abstimmung auf globaler Ebene. Für die geschilderten Umweltprobleme gibt es keine einfache Lösung. Der gelegentlich geäußerte Gedanke eines massiven Degrowth, also einer radikalen Schrumpfung der Wirtschaftsleistung (Jackson 2009), in jenen Ländern, die zurzeit vorrangig für die starke Umweltbelastung verantwortlich sind, stellt keine realistische Option da.

Während sich die Umweltbedingungen in den letzten Jahrzehnten konstant verschlechterten, haben wir gleichzeitig eine Entwicklung erlebt, die in bislang historisch einmaliger Weise dazu geführt hat, dass große Teile der Weltbevölkerung ext- 
remer Armut entkommen sind und deutlich bessere Lebensbedingungen vorfinden als sämtliche Generationen vor ihnen. Zugang zu Wasser, Bildung, Nahrung, medizinischer Versorgung - all dies hat sich rapide verbessert. Während 1990 beispielsweise $36 \%$ der Weltbevölkerung noch in extremer Armut lebten (1,9 Milliarden), so waren es im Jahr 2015 nur noch 730 Millionen (9,9\%) (World Bank 2019). Diesen Wohlstandsfortschritt zu erhalten und fortzusetzen, ist das wichtigste Ziel für die meisten Länder auf dieser Welt. Alle Bemühungen, Umweltprobleme zu reduzieren, müssen dies mit einbeziehen. Die wirtschaftliche und soziale Entwicklung wird den Druck auf die Umwelt absehbar deutlich erhöhen, selbst ohne ein weiteres Bevölkerungswachstum. Wir benötigen also neue Antworten darauf, wie ein hohes Maß an materiellem und sozialem Wohlstand erreicht werden kann, ohne dass damit die natürlichen Lebensgrundlagen für die Menschheit zerstört werden.

Die klassische Umweltpolitik reicht an dieser Stelle nicht mehr aus. Politikansätze, die einem end-of-pipe-Ansatz folgen und lediglich darauf abzielen, die Symptome einer umweltschädlichen Wirtschaftsweise zu kurieren, werden nicht genügen, um die bereits heute gravierenden Probleme und die sich deutlich verschlechternde Umweltsituation in den Griff zu bekommen. Notwendig sind grundlegende Transformationen der gesellschaftlichen und wirtschaftlichen Organisation. Neben der Energiewende sind weitere umfassende Wende-Prozesse erforderlich: eine Agrarwende, eine Chemiewende, eine Verkehrswende, eine Finanzwende et cetera. Diese Transformationen sind nur möglich, wenn es dazu begleitende Politiken gibt, die diese fördern, Akzeptanz schaffen, die entsprechenden Rahmenbedingungen setzen und die notwendigen Anstöße verleihen. Dass das 21. Jahrhundert ein Zeitalter der technischen, sozialen und ökologischen Wenden wird, kann man mit Sicherheit vorhersagen. Fraglich ist, ob diese erfolgreich sein werden. Grundsätzlich erforderlich ist in jedem Fall, dass die Gesellschaften transformationsfähiger werden. Gebraucht wird ein langer und sehr umfangreicher Transformationsprozess, der strukturiert und organisiert abläuft.

In diesem Zeitalter der Transformationen wird Deutschland eine Vorreiterrolle einnehmen müssen. In nur wenigen Ländern sind wie hier das entsprechende Wissen und die notwendigen Ressourcen vorhanden. Gleichzeitig verfügt Deutschland über die notwendige Stabilität, um eine solche Politik zu konzipieren. Darüber hinaus besitzt Deutschland genügend Ausstrahlungskraft, damit erfolgreiche Wende-Politiken anschließend international als Vorbild gelten können. Nicht zu vergessen ist auch, dass Deutschland wie nur wenige andere Länder, Verursacher verschiedener globaler Umweltprobleme ist. Dies gilt nicht nur für den Ausstoß von Treibhausgasen, sondern ebenso für den deutschen Beitrag zum nationalen und internationalen Biodiversitätsverlust, zur unkontrollierten Produktion und Freisetzung von chemischen Substanzen, zu massiv überhöhten Stickstoffeinträgen in die Umwelt und zur weltweiten Plastikverschmutzung. Diese Rolle basiert nicht nur auf dem Export von in Deutschland hergestellten Produkten, son- dern ebenfalls auf dem Export ganzer Wirtschaftsmodelle und Strukturen. Erfolgreiche Industriesektoren wie etwa die Chemieindustrie dienten als strukturelles Vorbild für andere Länder und wurden häufig mitsamt ihrer umweltschädlichen Eigenschaften kopiert. Die entsprechenden Leitbilder und auf Ressourcennutzung und end-of-pipe-Technologien zur Emissionsminderung basierende Produktionsprozesse wurden implizit weltweit verbreitet. Künftig ist es daher erforderlich, neue umweltverträgliche Konzepte und Denkweisen zu entwickeln und Strukturen zu schaffen, an denen sich erneut andere Länder orientieren können.

\section{Ausgangspunkt des Forschungsvorhabens evolution2green}

An dieser Stelle setzt das Vorhaben evolution2green (E2G) an, welches vom Bundesministerium für Bildung und Forschung gefördert wurde. Es verfolgte das Ziel der Entwicklung einer Gesamtstrategie für die Transformation des (deutschen) Wirtschaftssystems hin zu einer green economy sowie der Klärung von Hemmnissen und Erfolgsfaktoren für eine solche Entwicklung.

Im Rahmen dieses Vorhabens wurde zunächst ein Analyserahmen entwickelt, der auf drei unterschiedlichen konzeptionellen Ansätzen aufbaut: dem models-of-change-Ansatz (MoC), dem der evolutorischen Ökonomik entspringenden Ansatz der Pfadabhängigkeiten sowie einem Ansatz, der in den drei Leitstrategien Suffizienz, Effizienz und Konsistenz gründete. Darauf aufbauend wurde eine Anzahl von zentralen Problemfeldern einer Transformation in Richtung einer green economy analysiert und eine Analyse von ausgewählten Erfolgsfeldern durchgeführt. Für einzelne, strategisch bedeutsame Bereiche wurden Roadmaps für eine Transformation entwickelt.

Im Folgenden wird vor allem auf die Ergebnisse der Fallstudien von Erfolgsfällen eingegangen. Untersucht wurden hierbei Erfolgsfaktoren aus 20 Transformationsbeispielen in den Bereichen Wärme, Mobilität und Ressourcen. Diese Fallstudien wurden analysiert nach zentralen Erfolgsfaktoren, unter Berücksichtigung des Prozessverlaufs und unterschiedlicher Transformationstypen. Das angewandte Analysekonzept war der oben genannte MoC-Ansatz. Bei diesem Ansatz handelt es sich um ein Modell, das verschiedene Ansatzpunkte vereint: Vier Ebenen (Akteur/innen, Zeitaspekte, Veränderungsidee/ Lösungsvorschlag, Veränderungsprozess) beschreiben dabei die Bereiche, in denen durch aktives Handeln die Erfolgswahrscheinlichkeit für einen Veränderungsprozess gesteigert werden kann (Kristof 2010). Basierend auf diesem Modell wurden die der weiteren Untersuchung zugrunde liegenden Kernfaktoren identifiziert: Akteur/innen und ihre Qualifikation; Lösungsidee/Lösungsvorschlag; Zeitaspekte; Veränderungsprozesse; Pfadabhängigkeiten und Rahmenbedingungen.

Bei der durchgeführten Analyse wurden sowohl Transformationen in kleinen, klar abgegrenzten Bereichen betrachtet (bspw. kommunale Initiativen im Bereich der energetischen 
Gebäudesanierung) als auch Fallbeispiele mit größerer Reichweite (bspw. Elektromobilitätskonzepte in verschiedenen Ländern). Die untersuchten Transformationen waren dabei unterschiedlich weit entwickelt. Als Ergebnis konnte eine Anzahl übergreifender Faktoren identifiziert werden, die wichtige Erfolgsfaktoren für eine Transformation darstellen.

\section{Erfolgsfaktoren von Transformations- prozessen}

Zunächst konnte festgestellt werden, dass Landschaftsmerkmale und Rahmenbedingungen eine entscheidende Bedeutung für die Erfolgsaussichten einer Transformation haben. Erfolgreiche Transformationen außerhalb Deutschlands in den betrachteten Bereichen sind oftmals durch langjährig gewachsene Strukturen und akteursübergreifende Kooperation begünstigt. In den Niederlanden etwa sind regierungsgestützte Aktivitäten in den Bereichen Elektromobilität und Sanierung von Nullenergiehäusern auf eine jahrhundertealte korporatistische Kultur intersektoraler Kooperation und Kompromissfindung zurückzuführen.

Gleichzeitig ist ein freies Spielfeld, also die Abwesenheit von Pfadabhängigkeiten, ein entscheidender Faktor für erfolgreiche Transformationsprozesse. Im Falle Chinas stellte die Kombination aus bisher nur wenig erfolgreicher konventioneller Automobilindustrie sowie dem Anspruch Chinas, Weltmarktführer im neuen Bereich E-Mobilität zu werden, einen maßgeblichen Faktor zur erfolgreichen Transformation dar. Das Fehlen von markt- und systembeherrschenden Akteuren und von ihnen geprägten Pfadabhängigkeiten als positive Rahmenbedingung lässt sich auch in anderen Beispielen im E-Mobilitätssektor (Kalifornien, Niederlande und Norwegen) nachweisen.

Ein oft vorzufindendes Hindernis als Teil der allgemeinen Rahmenbedingungen ist der große Kostenvorteil bestehender Lösungen. Er ist meistens ein Ausdruck ökonomischer Pfadabhängigkeiten. Die Mehrheit der untersuchten Fallbeispiele belegt jedoch, dass durch gezielte Subventionierungen und Kostensenkungen in der Herstellung, also durch Eingriffe in die bestehenden Rahmenbedingungen, technologische und ökonomische Pfadabhängigkeiten zumindest teilweise ausgeglichen beziehungsweise überwunden werden können. Schwieriger ist es bei den nutzerbedingten Pfadabhängigkeiten, die auch Teil der allgemeinen Rahmenbedingungen sind. Nur geringe Veränderungen von Verhaltensweisen reichen für Transformationsprozesse kaum aus und größere Änderungen sind nur schwer zu erzielen.

Neben förderlichen Rahmenbedingungen ist die Existenz von sogenannten change agents von entscheidender Bedeutung. Change agents bilden sich oft als Außenseiter neben einem dominanten Regime heraus und spielen in allen untersuchten Fallbeispielen eine entscheidende Rolle in der erfolgreichen Implementierung einer nachhaltigen Idee. Sie agieren dabei als Promotor/innen, die einen Wandlungsprozess initiieren und gestalten. Entsprechend dem MoC-Ansatz, lässt sich eine
Kategorisierung in Fach-, Prozess-, Macht- und Beziehungspromotor/innen vornehmen. Eine weitere mögliche Kategorie von change agents lässt sich als „Problempromotor/innen“ bezeichnen. Hiermit sind Akteure gemeint, die vor allen anderen ein Problem erkennen und kontinuierlich auf dieses hinweisen.

Auffällig ist, dass Persönlichkeiten, die diese Transformationsprozesse einleiten, oftmals nicht dem Kern des etablierten Regimes entstammen, das sie verändern. Dennoch verfügen change agents notwendigerweise über die benötigten Ressourcen, etwa in Form von Fachwissen und Kontakten, um Rahmenbedingungen effektiv zu nutzen und Innovationen zum Durchbruch zu verhelfen. Dabei nehmen sie oftmals eine Brückenfunktion ein, die es ihnen erlaubt, sich mit anderen Akteuren zu vernetzen und zwischen den Stakeholdern zu vermitteln. Change agents können individuelle Persönlichkeiten in Unternehmen, wie Elon Musk im Falle von Tesla, oder aber auch Politiker/innen auf kommunaler und nationaler Ebene sein. Zuletzt können in manchen Fällen auch Institutionen als change agents verstanden werden, indem sie konkurrierende Anspruchsgruppen zusammenbringen, Zielkonflikte lösen und geeignetere Rahmenbedingungen für technologische Durchbrüche schaffen.

Gleichzeitig ließ sich anhand der Erfolgsbeispiele feststellen, dass das allgemeine Umweltbewusstsein und die zunehmende Beachtung der Probleme des Klimawandels zwar diskursiv eine große Rolle spielen, für das tatsächliche Gelingen von Transformationsprozessen jedoch konkret erfahrbare und dringliche Umweltprobleme von größerer Bedeutung sind. Als Beispiele dienen hier Ballungsgebiete in Kalifornien, Norwegen und China, die verstärkt mit Luftverschmutzung zu kämpfen hatten, was Anstrengungen hin zu umweltfreundlicheren Technologien verstärkte und den Weg für die erfolgreiche Etablierung des E-Mobilitätssektors ebnete.

Neben einer unmittelbar erfahrbaren Lösung für Umweltprobleme sind zusätzliche co-benefits entscheidende Erfolgsfaktoren für Transformationsprozesse. Diese betreffen beispielsweise ökonomische, aber auch sozialpolitische und gesundheitsrelevante Aspekte. So lässt sich feststellen, dass in von Unternehmen vorangetriebenen Transformationen wirtschaftlicher Erfolg meist den ausschlaggebenden Faktor für eine erfolgreiche Transformation darstellte. Auch im Rahmen von auf kommunaler Ebene ablaufenden Transformationsprozessen standen zumeist wirtschaftliche Aspekte sowie eine langfristige Sicherung der Lebensqualität im Zentrum der Initiativen. Aus den Untersuchungen ergibt sich, dass co-benefits für alle Transformationstypen relevant sind. Im Umkehrschluss stehen Transformationsprozesse vor erheblichen Herausforderungen, wenn sie im dominanten Regime nur wenige co-benefits versprechen.

Eine weitere Erkenntnis der Analyse betrifft den Aspekt, dass es einer passgenauen Lösung sowie iterativer Anpassung von Strategien bedarf, um eine erfolgreiche Transformation zu erreichen. Denn um Co-Benefits einer Transformation realisieren zu können, braucht es eine passgenaue Umsetzungslö- 
sung, die Umwelt- und Nachhaltigkeitszielstellungen mit wirtschaftlichen Interessen verknüpft. Eine solche Lösung wurde in den untersuchten Beispielen meist mithilfe einer mutigen Vision und Skalierungsstrategie umgesetzt. Insbesondere wurden relevante Stakeholdergruppen nacheinander eingebunden und deren spezifische Bedürfnisse berücksichtigt. Dabei wurden einzelne Zielgruppen individuell oder nacheinander adressiert, um Raum für Reaktionen und Anpassungen bei Fehlschlägen zu lassen. Damit sind Passgenauigkeit und Anpassungsfähigkeit von entscheidender Bedeutung für eine erfolgreiche Transformation.

Ein anderes Merkmal erfolgreicher Transformationen ist Reflexivität. Neue Erkenntnisse werden systematisch und dynamisch in die zugrunde liegende Strategie integriert. Diese Reflexivität lässt sich in unterschiedlichen Prozessen finden. Sich ändernde Rahmenbedingungen und Erfahrungen werden hierbei aufgenommen und führen $\mathrm{zu}$ einer entsprechenden Anpassung des Vorgehens.

Darüber hinaus waren Zeitaspekte wie spezielle Auslöser und Zeitfenster (windows of opportunity), die oftmals strategisch genutzt werden, in den meisten untersuchten Beispielen ausschlaggebend. Dabei lassen sich drei wichtige Arten von Zeitfenstern unterscheiden: Zum einen historische Ereignisse und Krisen, die zu größeren Umwälzungen führten. So konnten im Zuge der Ölkrise Change Agents den dänischen Heizungssektor umgestalten. Zum anderen politische und regulatorische Neuerungen wie neue Gesetze, Richtlinien und Verpflichtungen, die eine disruptive Wirkung entfalten können und gleichzeitig Akteur/innen Möglichkeiten für die Entwicklung neuer Geschäftsmodelle geben. Eine dritte Art von Gelegenheitsfenstern sind disruptive technologische Innovationen, wobei technologische Neuerungen die Entwicklung neuer Lösungen und die Verdrängung etablierter Stakeholder erlauben. So hat beispielsweise die Entwicklung leistungsfähigerer Lithium-Ionen-Akkus die Entwicklung der E-Mobilität entscheidend beeinflusst.

Ein weiterer wichtiger Erfolgsfaktor für Transformationsprozesse besteht in gezielter selektiver Koordination. So ließ sich im Rahmen der Analyse feststellen, dass horizontaler Koordination zwischen Akteur/innen aus Politik, Wirtschaft, Zivilgesellschaft und Wissenschaft eine vergleichsweise hohe Bedeutung zukommt. Koordination wirkt sich dann positiv aus, wenn zur Initiierung des Transformationsprozesses unterschiedliche Arten von Wissen und Kompetenzen benötigt werden oder wenn politische Mehrheiten für gesetzgeberische Änderungen beschafft werden müssen. Während zu Beginn eines Transformationsprozesses oftmals die Koordination in der Nische oder zwischen verschiedenen Nischen genügt, ist mit zunehmender Ausdehnung eine Vielzahl von Akteur/innen von der Transformation betroffen sowie für deren Realisierung als Aktive notwendig. Am erfolgreichsten ist Koordination, wenn es gelingt, gruppenübergreifende Koalitionen zu bilden.

Neben der Koordination relevanter Akteure werden für erfolgreiche Transformationen auch ausreichende Ressourcen benötigt. Während zur Initiierung von Nischenaktivitäten wie dem Aufbau von Repair Cafés in lokalen Gemeinschaften oder verpackungsfreiem Einkaufen oftmals keine oder nur geringe finanzielle Ressourcen benötigt werden, macht die Umgestaltung größerer sozio-technischer Systeme und ganzer Sektorentwicklungen hingegen umfangreiche Investitionen erforderlich (UNEP 2011). Beispiele sind die Umgestaltung des auf dem Verbrennungsmotor basierenden Individualverkehrs, die Neuausrichtung des gängigen Produktdesigns hin zu höherer Kreislaufwirtschaftsfähigkeit oder eine Erneuerung unzureichend gedämmter Gebäude und fossiler Heizungssysteme. Trotz weitreichender öffentlicher Finanzierungsmöglichkeiten wird aus den betrachteten Beispielen auch deutlich, dass für eine vollständige Transformation in großen Sektoren umfangreiche private Investitionen erforderlich sind. Das bedeutet im Umkehrschluss, dass privatwirtschaftliche Akteure mit signifikanten Ressourcen die Transformation vorantreiben oder diese durch Koalitionsbemühungen als Unterstützer/innen gewonnen werden müssen. Dies setzt wiederum voraus, dass in einem hohen Maße Vertrauen in den Transformationsprozess geschaffen werden kann.

Aus den hier dargestellten Erkenntnissen bezüglich der Erfolgsfaktoren von Transformationen lässt sich eine Reihe von politischen Handlungsempfehlungen ableiten. Dabei wird deutlich, dass Transformationen der politischen Gestaltbarkeit zugänglich sind. Hierfür werden im Folgenden verschiedene Ansätze aufgezeigt.

\section{Aktives Stakeholder-Management}

Aus umweltpolitischer Sicht ist entscheidend, dass sowohl das Vorhandensein von change agents als auch das Zusammenspiel von Akteurskonstellationen im Rahmen eines Stakeholder-Managements gezielt beeinflusst werden können: Change agents können aufgebaut werden und ihre Vernetzung untereinander kann entscheidend gefördert werden. Ein Beispiel für ein aktives Stakeholder Management, das auch in den gesellschaftlichen Bereich hinausgreifen kann, ist die des Europäischen Dachverbandes der Sustainable Investment Foren (Eurosif). Der Verband wurde um die Jahrtausendwende konkret auf Anregung der Europäischen Kommission gegründet und hat in seinen ersten Jahren einige finanzielle und politische Unterstützung bei seinem Aufbau durch die Generaldirektion für Beschäftigung, Soziales und Integration erhalten. Auf diese Weise hat die Kommission einen Gegenspieler zu den rein an finanziellen Interessen ausgerichteten klassischen Verbänden der Finanzindustrie geschaffen. Gleichzeitig wurde damit auch ein Anstoß gegeben, auf nationaler Ebene weitere solcher Verbände zu gründen, die inzwischen vielerorts eine wichtige Rolle bei der Transformation des Finanzwesens in Richtung von mehr Nachhaltigkeit spielen.

Stakeholder-Management im Rahmen einer transformativen Umweltpolitik bedeutet mithin nicht nur, change agents zu identifizieren und sich mit ihnen auszutauschen. Ein aktives 
Stakeholder Management kann darüber deutlich hinausgehen. Es identifiziert Lücken und Schwachstellen im Akteursgefüge und ergreift dann Maßnahmen, diese Lücken zu füllen beziehungsweis die Schwachstellen zu stärken, indem gemeinsam mit anderen gesellschaftlichen Akteuren geeignete Initiativen ergriffen werden.

\section{Management von Windows of Opportunity}

In der Vergangenheit wurden Gelegenheitsfenster oftmals nicht oder nur unzureichend genutzt. Für eine transformative Umweltpolitik ist es wichtig, Gelegenheitsfenster frühzeitig, also möglichst im Vorfeld, zu identifizieren. Auf diese Weise kann man sich bereits vorbereiten, Lösungsvorschläge entwickeln, andere Akteure frühzeitig einbinden und Ansatzmöglichkeiten schaffen, um das Thema in den Politikstrom aufzunehmen (Wolff et al. 2018).

Jenseits dieser Prozesse sollte das Management von Gelegenheitsfenstern nicht nur in der Vorbereitung auf unabhängig von der Politik eintretenden Ereignissen bestehen. Eine transformative Umweltpolitik muss dynamisch vorgehen und neue Gelegenheitsfenster schaffen. Dies ist zunächst in einfachem Maße möglich, indem wiederkehrende Gelegenheitsfenster in den Politikprozess integriert werden. Denn solche Fenster haben nicht nur als Auslöser einer Transformation eine entscheidende Bedeutung, sondern werden im weiteren Verlauf des Transformationsprozesses gebraucht, um dessen Fortschritt zu gewährleisten und den Prozess in die gewünschte Richtung zu lenken. Diese Art von wiederauftretenden Gelegenheitsfenstern lässt sich unter anderem durch fest verankerte regelmäßige Review- und Monitoringprozesse umsetzen. Transformative Umweltpolitik kann aber noch einen Schritt weitergehen und selbst Auslöser von Gelegenheitsfenstern sein. So sind zum Beispiel die umweltbezogenen Probleme im Verkehrs-, Agrar- und Chemiesektor vielfältig und gravierende Problemlagen einschließlich deutlicher Gesetzverstöße sind hier zahlreich. Eine in die Gesellschaft wirkende Umweltpolitik muss sich nicht darauf beschränken, dass andere Akteure eigeninitiativ diesen Problemen nachgehen und sie in die gesellschaftliche Diskussion bringen.

\section{Wertewandel}

Eine grundlegende Voraussetzung für die Transformation zu einer green economy wird ein umfassender Wertewandel sein. Dieser findet aber nicht unabhängig von politischen Einflüssen statt. So ist es bereits in der Vergangenheit gelungen, bestimmtes Verhalten durch politisches Eingreifen mit einem schlechten Image zu belegen. Ein praktisches Beispiel ist die Kommunikation von Nichtraucherschutz als wesentlicher Vorteil einer Transformation zu einer raucherfreien Gesellschaft. Anstatt nur auf die Gesundheitsschäden für Raucher/innen selbst hinzuweisen, wurde der Aspekt der Schädigung Unbeteiligter neu eingeführt und durch Informationskampagnen in den Vorder- grund der Debatte gerückt. Damit bekam der Übergang zu einer rauchfreien Gesellschaft einen essenziellen neuen Nutzen und wurde von weiten Teilen der Bevölkerung mitgetragen. Ein ähnlicher Prozess lässt sich hinsichtlich der Regulierung und der staatlichen Kommunikation zur Pkw-Nutzung in Singapur feststellen. Hier existieren bereits seit Längerem rigide Auflagen für die Anschaffung und Nutzung privater Pkws. Zusätzlich verfolgt die Regierung nun das Ziel, die schädigenden Aspekte des Autofahrens für weite Teile der Gesellschaft aufzuzeigen und damit das Image des Autos beziehungsweise des Autofahrenden zu verändern. Es ist diese Art der Mitgestaltung von gesellschaftlichen Wertvorstellungen, die eine transformative Umweltpolitik benötigt.

Eine ähnliche Rolle für eine transformative Umweltpolitik lässt sich im Zusammenhang mit co-benefits, also zusätzlichen Vorteilen von Transformationsprozessen, feststellen. Co-benefits können von staatlicher Seite zwar oftmals nicht direkt kreiert werden. Aber der Staat hat einigen Einfluss auf die gesellschaftliche Wahrnehmung, was einen Zusatznutzen darstellt. Diese Wahrnehmung ist der politischen Gestaltbarkeit durchaus zugänglich. Was als co-benefit gilt, ist nicht zuletzt eine Frage der individuellen Perzeption und an dieser Stelle verfügt der Staat über zahlreiche Möglichkeiten, auf eine bestimmte Wahrnehmung hinzuwirken. Dabei spielt die Erkenntnis eine wichtige Rolle, dass Werte und Bedürfnisse, an die Transformationen Anschluss suchen und die die Wahrnehmung von co-benefits maßgeblich mitbestimmen, nicht statisch im derzeitigen Zustand verbleiben (Grimm et al. 2014).

\section{Verlässlichkeit im Wandel}

Wie bereits erläutert, braucht es für umfangreiche Transformationen großskalige Investitionen. Ein guter Teil der benötigten Investitionen wird durch die Privatwirtschaft getragen werden müssen und hier gilt, dass eine Transformation hin zu einer green economy zunächst Unsicherheit schafft und damit mögliche Investoren abschreckt. Damit sich Investoren auf die Bereitstellung dieser Mittel einlassen, brauchen sie eine gewisse Planungssicherheit. Grundlegend für die Planungssicherheit sind zum einen entsprechende strategische Entscheidungen und Strategiefestlegungen. Zum anderen und eng verbunden mit der Strategieentwicklung geht es um die Gestaltung von neuen Pfadabhängigkeiten, etwa durch die Schaffung neuer Netzwerke, rechtlicher Festlegungen et cetera. Systemdenken muss in der Politikgestaltung stärker durchgesetzt werden, um das Entstehen von Pfadabhängigkeiten möglichst bewusst zu gestalten.

\section{Gerechtigkeit als Grundlage sozial-ökologischer Transformationen}

In den kommenden 50 Jahren brauchen wir radikale Umbrüche in praktisch allen Wirtschaftssektoren. Für die in diesem Artikel beschriebene sehr weit greifende Rolle von transforma- 
tiver Umweltpolitik in diesem Prozess ist gesellschaftliche Legitimation entscheidend. Eine transformative Umweltpolitik wird sich für ihre Aktivitäten noch mehr rechtfertigen müssen, als dies in der Vergangenheit für die Umweltpolitik insgesamt schon der Fall war. Entscheidend wird sein, Gerechtigkeit in den Transformationsprozessen explizit zu machen, neu auszuhandeln und dann herzustellen. Diese Gerechtigkeitsfrage wird in den nächsten Jahren vermutlich eine zentrale Frage der sozial-ökologischen Transformation werden.

Ein wichtiger Aspekt ist dabei unzweifelhaft die soziale Gerechtigkeit. Die Gefahr besteht permanent, dass jene Bevölkerungsschichten, die über die geringsten Ressourcen verfügen, unter den Transformationen besonders leiden. Dies ist, wie die fortschreitende Energiewende zeigt, zwar eine ganz wesentliche, aber keineswegs die einzige Dimension von Gerechtigkeit im sozial-ökologischen Transformationsprozess. Eine zweite, ebenso wichtige, jedoch schwieriger zu fassende Dimension ist die der Verlierer/innen und Gewinner/innen im Transformationsprozess. Gerade hier wird die Behandlung von Gerechtigkeitsaspekten entscheidend sein. Eine sozial-ökologische Transformation wird nicht gelingen, wenn alle Verlierer des Transformationsprozesses sich als Benachteiligte wahrnehmen, dies gesellschaftlich auch akzeptiert wird und die Kosten der Transformationen dann durch Kompensationszahlungen erheblich erhöht werden. Eine gerechte sozial-ökologische Transformation setzt voraus, ein gesellschaftliches Verständnis darüber zu erzielen, dass viele der Benachteiligten der Transformation, sei es etwa im Energie-, Verkehrs-, Agrar- oder Chemiesektor, die Privilegierten der Vergangenheit waren. Die Beschneidung ihrer Möglichkeiten ist nicht ein unbilliger Eingriff in ihre Rechte, sondern das Herstellen von Gerechtigkeit in einer Welt, in der wachsende Umweltrisiken von wenigen verursacht und Umweltschäden von vielen getragen werden.

\section{Literatur}

Grimm, F./Heinrichs, H./Neumann, K. (2014): Entwicklung eines Integrated Assessment Modells: Nachhaltige Entwicklung in Deutschland. Band 1: Hauptband. Dessau-Roßlau, Umweltbundesamt.

Jackson, T. (2009): Prosperity Without Growth. Economics For A Finite Planet. London, Earthscan.

Kristof, K. (2010): Models of Change: Einführung und Verbreitung sozialer Innovationen und gesellschaftlicher Veränderungen in transdisziplinärer Perspektive. Zürich, vdf Hochschulverlag.

Rockström, J. et al. (2009): Planetary Boundaries: Exploring the Safe Operating Space for Humanity. In: Ecology and Society 14/2, 32.

UNEP (2011): Towards a Green Economy: Pathways to Sustainable Development and Poverty Eradication. Paris, United Nations Environment Programme.

UNEP (2019): Global Environment Outlook - GEO-6: Healthy Planet, Healthy People. Nairobi, United Nations.

Wolff, F./Heyen, D. A./Brohmann, B./Grießhammer, R./Jakob, K./Graaf, L. (2018): Transformative Umweltpolitik: Nachhaltige Entwicklung konsequent fördern und gestalten. Ein Wegweiser für den Geschäftsbereich des BMU. Dessau-Roßlau, Umweltbundesamt.

World Bank (2019): Poverty Headcount Ratio at \$1.90 a Day (2011 PPP) (\% of population). www.data.worldbank.org/indicator/SI.POV.DDAY

\section{AUTOR/INNEN + KONTAKT}

Walter Kahlenborn ist Mitbegründer und Geschäftsführer von adelphi research und adelphi consult.

Linda Hölscher arbeitet in den Themenbereichen Energie, Green Economy, Emissionshandel und Anpassung an den Klimawandel bei adelphi.

Daniel Weiß leitet bei adelphi Vorhaben auf nationaler und internationaler Ebene zu den Themen Nachhaltigkeitsstrategien in Politik und Wirtschaft, Green Economy und Umweltmanagement.

adelphi research gGmbH/adelphi consult $\mathrm{GmbH}$, Alt-Moabit 91, 10559 Berlin. Tel: +49 30890006840 E-Mail: kahlenborn@adelphi.de, hoelscher@adelphi.de, weiss@adelphi.de, Website: https://www.adelphi.de/de
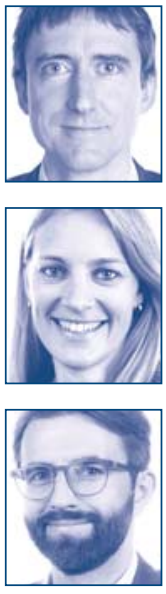

\section{Auf der SUCHE nach dem passenden GESCHENK?}

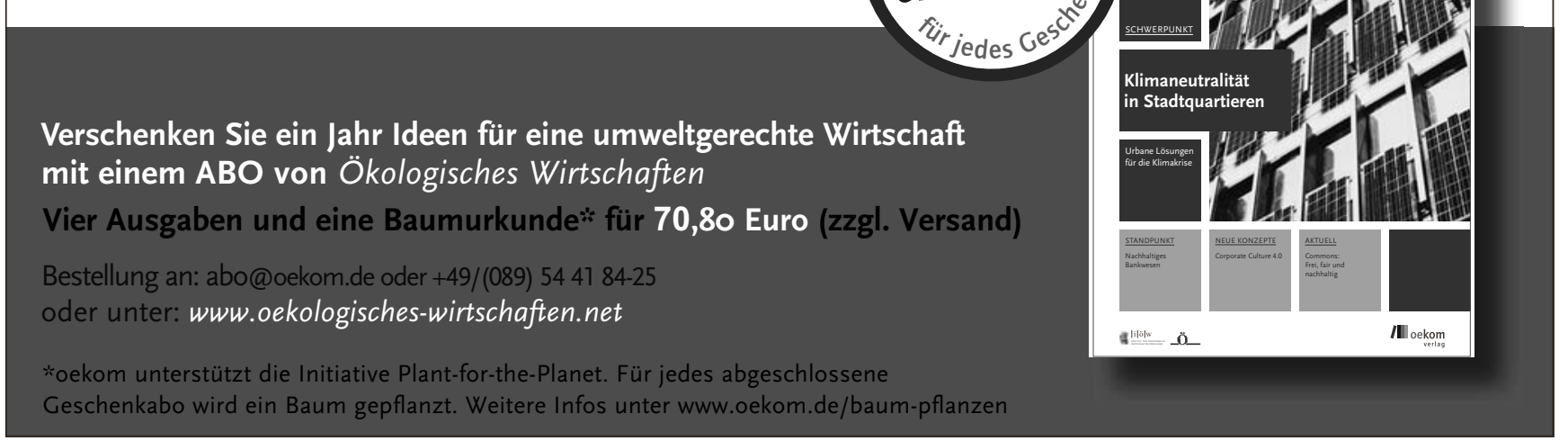

